\title{
A MIMO Radar System based on Fractal Antenna Arrays for Level Measurement Applications
}

\author{
Christoph Dahl ${ }^{1}$, Michael Vogt ${ }^{2}$, and Ilona Rolfes ${ }^{1}$ \\ ${ }^{1}$ Institute of Microwave Systems, Ruhr University Bochum, 44801 Bochum, Germany \\ ${ }^{2}$ Institute of Electronic Circuits, Ruhr University Bochum, 44801 Bochum, Germany \\ Correspondence: Christoph Dahl (christoph.dahl@rub.de)
}

Received: 11 December 2020 - Revised: 26 February 2021 - Accepted: 12 March 2021 - Published: 17 December 2021

\begin{abstract}
In this contribution, the design of a multiple-input multiple-output (MIMO) radar system in $77-81 \mathrm{GHz}$ range with 18 transmitting antennas and 24 receiving antennas for measuring the height profile of bulk solids in silos, is presented and discussed. The antenna array topologies are optimized by utilizing space filling fractals in order to approximate a circular shaped antenna array on a hexagonal grid. The proposed MIMO radar system achieves an angular resolution of $3.1^{\circ}$ for a maximum scanning angle of $\pm 45^{\circ}$ and a side lobe suppression of $12.6 \mathrm{~dB}$. The performance of the system has been evaluated by test measurements on a sand heap, showing an improved measurement accuracy compared to conventional radar level systems.
\end{abstract}

\section{Introduction}

In the context of level measurement, determining the volume of bulk solids in silos by means of radar is a challenging task, due to the complex spatial structure of the bulk solid heap (Brooker et al., 2006; Nienhaus et al., 2009). The MIMO radar concept is utilized in a variety of $3 \mathrm{D}$ imaging scenarios, like whole-body imaging or remote sensing (Ahmed et al., 2009; Ender and Klare, 2009; Klare et al., 2010). Since integrated radar chips are emerging in automotive applications, MIMO radar systems are appropriate to improve the accuracy of height profile measurements of bulk solids by realizing scanning radar systems using digital beam forming (Dahl et al., 2017a; Nienhaus et al., 2007; Zankl et al., 2015). As the performance of a MIMO radar system is mainly limited by the number of transmit and receive channels, the topology of the transmit and receive antenna array are an important factor. Fractal antenna arrays can ad- vantageously be used to optimize the angular resolution and side lobe level of a MIMO radar system. This is achieved by combining sub arrays based on space-filling fractals in order to approximate circular shaped arrays on a hexagonal grid (Dahl et al., 2017b; Werner et al., 2004). In the work presented here, fractal arrays have been adapted for realizing MIMO radar systems and the developed concept has been used and validated for radar level measurement applications.

\section{Fundamentals}

In the following, time-division multiplexed MIMO radar systems with spatially separated antenna arrays for transmit and receive are considered. The angular resolution $\Delta \theta$ of a MIMO radar system depends on the two-way radiation pattern which is given by the virtual array factor $\mathrm{AF}_{\mathrm{V}}$, expressed in spherical coordinates using the polar angle $\theta$ and the azimuthal angle $\varphi$. It can be obtained by multiplying the array factor $\mathrm{AF}_{\mathrm{Tx}}$ of the transmitting array with the array factor $\mathrm{AF}_{\mathrm{Rx}}$ of the receiving array (Fishler et al., 2004):

$\operatorname{AF}_{\mathrm{V}}(\theta, \varphi)=\operatorname{AF}_{\mathrm{Tx}}(\theta, \varphi) \cdot \operatorname{AF}_{\mathrm{Rx}}(\theta, \varphi)$.

As illustrated in Fig. 1, the virtual array factor $\mathrm{AF}_{\mathrm{V}}$ of the MIMO radar corresponds to a virtual antenna array, which is given by the convolution of the transmitting array with the receiving array (Fishler et al., 2004). The radar echo with each transmit-receive combination is assigned to one virtual antenna element. By applying two-dimensional digital beam forming and a range compression to the echo data represented in the virtual antenna array, a 3D radar image is reconstructed in spherical coordinates. After interpolation to Cartesian coordinates, the surface profile of the bulk solid 


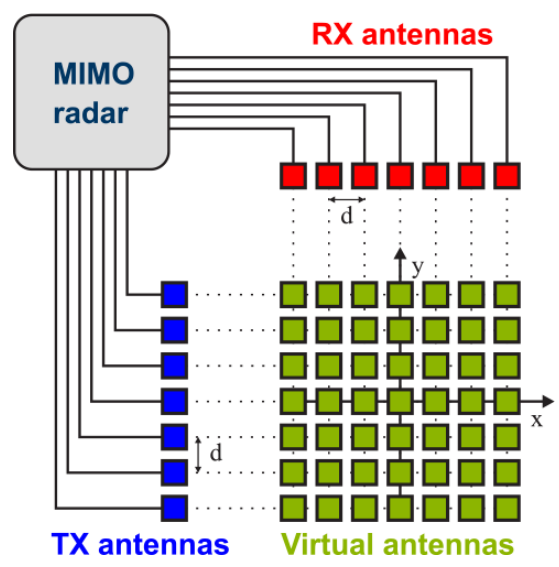

Figure 1. MIMO radar concept: Virtual array formed by the convolution of the transmit array and the receive array.

heap can be reconstructed and the filling volume can be measured (Dahl et al., 2015a).

In general, the array factor AF of a planar antenna array, consisting of $N$ discrete elements, depends on the element positions $\left(x_{n}, y_{n}\right)$, the complex element weights $w_{n}$, and the wave number $k=2 \pi f / c$, with the frequency $f$ of the radiated electromagnetic waves (Volakis, 2007). The polar and azimuthal angles $\theta$ and $\varphi$,respectively, are transformed into $u / v$-coordinates:

$u=\cos \theta \cdot \cos \varphi$

$v=\cos \theta \cdot \sin \varphi$.

The array factor AF can then be written as:

$\operatorname{AF}(\theta, \varphi)=\sum_{n=1}^{N} w_{n} \cdot e^{j \cdot k \cdot\left(x_{n} \cdot u+y_{n} \cdot v\right)}$.

In order to steer the main lobe to a direction $\left(u_{0}, v_{0}\right)$, the complex weights $w_{n}$ in the array factor AF have to be chosen as follows:

$w_{n}=\frac{1}{N} \cdot e^{-j \cdot k \cdot\left(x_{n} \cdot u_{0}+y_{n} \cdot v_{0}\right)}$.

In the following, all array factors are analyzed with the main lobe steered to $u_{0}=v_{0}=0$.

For a MIMO radar system with given numbers of transmit and receive antennas $N_{\mathrm{Tx}}$ and $N_{\mathrm{Rx}}$, respectively, the virtual array with $N_{\mathrm{V}}=N_{\mathrm{Tx}} \cdot N_{\mathrm{Rx}}$ virtual antenna elements can be optimized by approximating a circular shaped array on a hexagonal grid in order to maximize the virtual aperture diameter (Dahl et al., 2015b). As a result, the side lobe suppression SLS and the angular resolution $\Delta \theta$ of the virtual array factor $\mathrm{AF}_{\mathrm{V}}$ are improved, compared to conventional MIMO radar configurations based on perpendicular linear antenna arrays (Dahl et al., 2015b; Zhuge and Yarovoy, 2012). In the following, space filling Fudgeflake and Gosper island fractals on a hexagonal grid are utilized for this purpose (Dahl et al., 2016, 2017b).

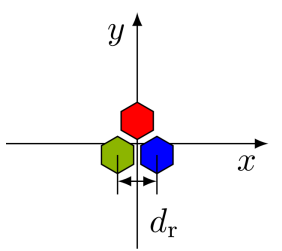

(a)

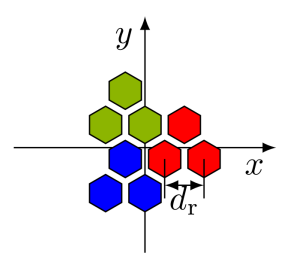

(b)

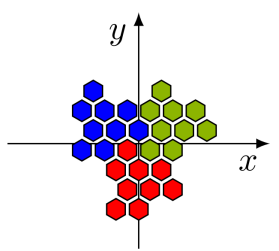

(c)
Figure 2. Fudgeflake fractal: (a) first, (b) second, and (c) third stage.

\subsection{Fudgeflake fractal}

As shown in Fig. 2a, the first stage $(L=1)$ of the Fudgeflake fractal can be used to combine three antenna elements to a regular triangle on a hexagonal grid (Werner et al., 2004).

The corresponding array factor $\mathrm{AF}_{\mathrm{F}, 1}$ can be written as follows using the radial element spacing $d_{\mathrm{r}}$ :

$\operatorname{AF}_{\mathrm{F}, 1}\left(\theta, \varphi, d_{\mathrm{r}}\right)=\frac{1}{\sqrt{3}} \cdot \sum_{n=1}^{3} e^{j \cdot k \cdot \frac{d_{\mathrm{r}}}{\sqrt{3}} \cdot \sin (\theta) \cdot \sin \left(\varphi-\frac{n \pi}{3}\right)}$.

As illustrated in Fig. 2, higher stages of the fractal can be formed by combining first stages of the fractal in a selfsimilar way (Dahl et al., 2017b). The array factor $\mathrm{AF}_{\mathrm{F}, L}$ of the fractal array in a stage $L$ can be expressed by the product of scaled an rotated replicas of the first stage array factor $\mathrm{AF}_{\mathrm{F}, 1}$ :

$\operatorname{AF}_{\mathrm{F}, L}\left(\theta, \varphi, d_{\mathrm{r}}\right)=\prod_{l=1}^{L} \mathrm{AF}_{\mathrm{F}, 1}\left(\theta, \varphi_{\mathrm{F}, l-1}, d_{\mathrm{F}, l-1}\right)$

The rotation angle $\varphi_{\mathrm{F}, l}^{\prime}$ of the array is proportional to the stage $l$ :

$\varphi_{\mathrm{F}, l}=\varphi-\varphi_{\mathrm{F}, l}^{\prime}$

$\varphi_{\mathrm{F}, l}^{\prime}=-l \cdot \frac{\pi}{6}$.

The scaling of the element spacing $d_{\mathrm{r}}$ increases with increasing number of elements $N_{\mathrm{F}, l}$ of the corresponding stage $l$ :

$$
\begin{aligned}
d_{\mathrm{F}, l} & =d_{\mathrm{r}} \cdot \sqrt{N_{\mathrm{F}, l}} \\
N_{\mathrm{F}, l} & =3^{l} .
\end{aligned}
$$

\subsection{Gosper island fractal}

According to Fig. 3a, the first stage of the Gosper island fractal combines six antenna elements to a regular hexagon with an additional element in the center (Werner et al., 2003).

The corresponding array factor $\mathrm{AF}_{\mathrm{G}, 1}$ can be written as:

$$
\mathrm{AF}_{\mathrm{G}, 1}\left(\theta, \varphi, d_{\mathrm{r}}\right)=\frac{1}{\sqrt{7}} \cdot \sum_{n=1}^{6} e^{j \cdot k \cdot d_{\mathrm{r}} \cdot \sin (\theta) \cdot \cos \left(\varphi-\frac{n \pi}{6}\right)}+\frac{1}{\sqrt{7}} .
$$




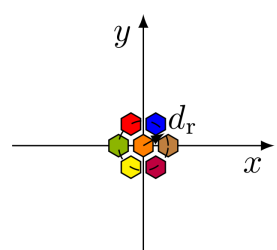

(a)

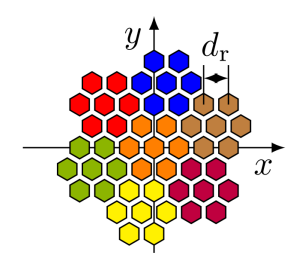

(b)

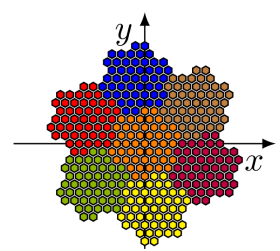

(c)
Figure 3. Gosper island fractal: (a) first, (b) second, and (c) third stage.

The higher stages of the Gosper island fractal can be created by combining multiple first stages of the fractal, see Fig. 3 (Dahl et al., 2017b). In analogy to Eqs. (7)-(11), the array factor $\mathrm{AF}_{\mathrm{G}, L}$ in a stage $L$ of the Gosper island fractal can be written as:

$\mathrm{AF}_{\mathrm{G}, L}\left(\theta, \varphi, d_{\mathrm{r}}\right)=\prod_{l=1}^{L} \mathrm{AF}_{\mathrm{G}, 1}\left(\theta, \varphi_{\mathrm{G}, l-1}, d_{\mathrm{G}, l-1}\right)$,

with

$$
\begin{aligned}
\varphi_{\mathrm{G}, l} & =\varphi-\varphi_{\mathrm{G}, l}^{\prime} \\
\varphi_{\mathrm{G}, l}^{\prime} & =l \cdot \arctan \left(\frac{\sqrt{3}}{5}\right) \\
d_{\mathrm{G}, l} & =d_{\mathrm{r}} \cdot \sqrt{N_{\mathrm{G}, l}} \\
N_{\mathrm{G}, l} & =7 .
\end{aligned}
$$

In addition, both fractals can be combined to construct MIMO radar systems with circularly shaped virtual antenna arrays on a hexagonal grid (Dahl et al., 2017b). In order to avoid grating lobes, the element spacing $d_{\mathrm{r}}$ in the virtual array has to be chosen according to the minimum wavelength $\lambda_{\min }$ and the maximum steering angle $\theta_{\max }$ :

$d_{\mathrm{r}}=\frac{2}{\sqrt{3}} \cdot \frac{\lambda_{\min }}{1+\sin \left(\theta_{\max }\right)}$.

\section{Realized MIMO radar system}

A MIMO radar system with six Texas Instruments IWR1443 MIMO radar chips, each providing 3 transmit and 4 receive channels, has been realized, see Fig. 4 , resulting in $N_{\text {Tx }}=18$ transmit antennas and $N_{\mathrm{Rx}}=24$ receive antennas.

In the chips the frequency modulated continuous wave (FMCW) radar concept is realized. Time-division multiplexing is utilized for all 18 transmit channels and the receive channels are operated simultaneously. With a chirp duration of $194.1 \mu \mathrm{s}$ the data acquisition for all transmit and receive combinations can be achieved in $8 \mathrm{~ms}$. The measurement rate of the demonstrator system is limited by the data transfer and the signal processing on a personal computer to approximately 4 min and can be further improved by utilizing digital

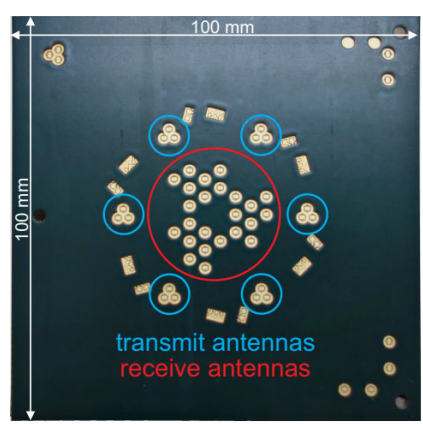

(a)

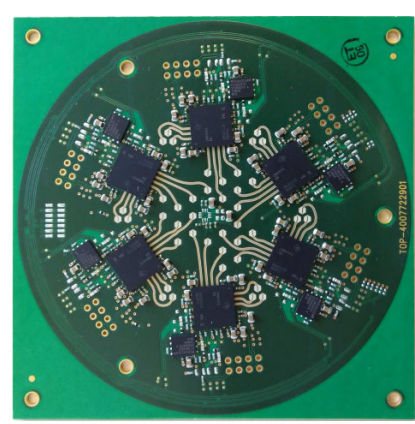

(b)
Figure 4. Realized MIMO radar system: (a) Antenna elements on the front side and (b) MIMO radar chips on the back side.

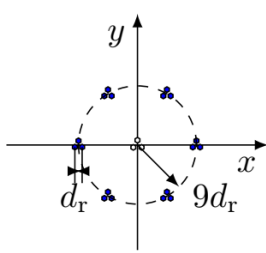

(a)

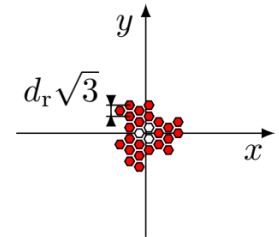

(b)

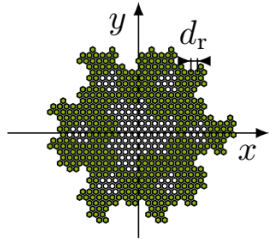

(c)
Figure 5. Fractal MIMO concept: (a) Transmitting array, (b) receiving array, and (c) corresponding virtual array. The white elements have been removed in order to adjust the number of transmit- and receive antennas.

signal processors. By operating in the $77-81 \mathrm{GHz}$ frequency range, a range resolution $\Delta R=37.5 \mathrm{~mm}$ is achieved. Assuming a homodyne receiver architecture, the maximum unambiguous range of $R_{\mathrm{ua}}$ is given by the number of complex samples $N_{\mathrm{IF}}=1024$ for each intermediate frequency signal and the range resolution $\Delta R$ :

$R_{\mathrm{ua}}=N_{\mathrm{IF}} \cdot \Delta R=38.4 \mathrm{~m}$.

Aperture coupled patch antenna array elements fed by micro strip lines with an element gain $G_{\mathrm{E}}=8.2 \mathrm{dBi}$ have been realized (Buck and Pozar, 1986). Each of the $N_{\mathrm{V}}=N_{\mathrm{Tx}} \cdot N_{\mathrm{Rx}}=$ 432 virtual antenna elements corresponds to a specific transmit and receive combination.

\subsection{Fractal array topology}

The concept of fractal antenna arrays has been applied for the design of a compact circularly shaped virtual array on a hexagonal grid with an aperture diameter of $50 \mathrm{~mm}$. An antenna spacing $d_{\mathrm{r}}=2.5 \mathrm{~mm}$ is used to allow for a maximum steering angle $\theta_{\max }=45^{\circ}$, according to Eq. (18). As shown in Fig. 5a, a combination of the first stage of the Fudgeflake fractal and the first stage of the Gosper island is used for the transmitting array. The antenna elements at the center have been removed in order to allow for a compact alignment with the receiving array, as shown in Fig. 4a. 


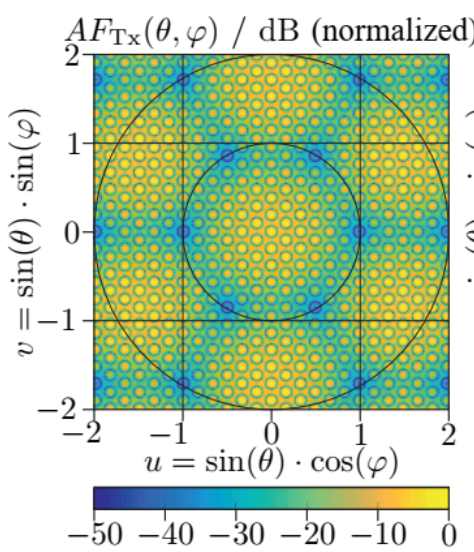

(a)

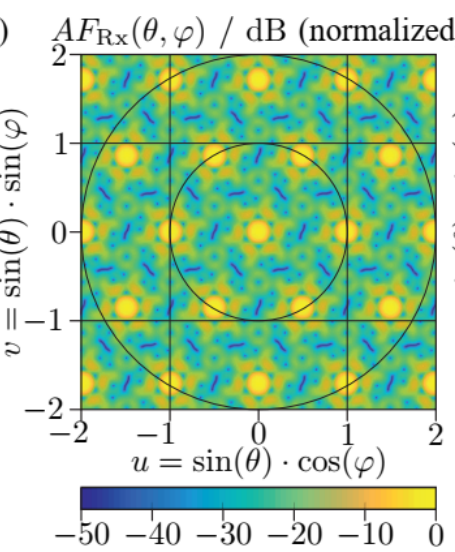

(b)

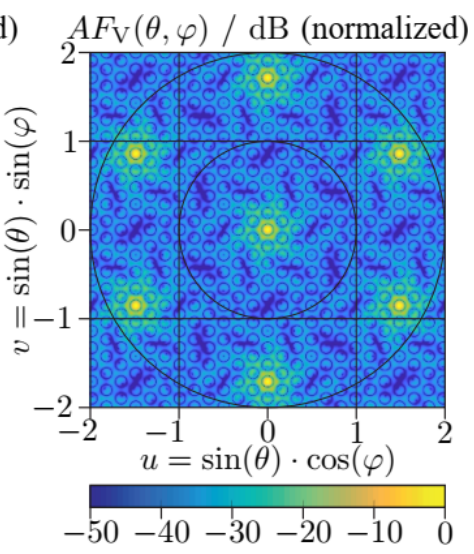

(c)

Figure 6. Fractal MIMO concept: (a) Transmitting array factor, (b) receiving array factor, and (c) virtual array factor.

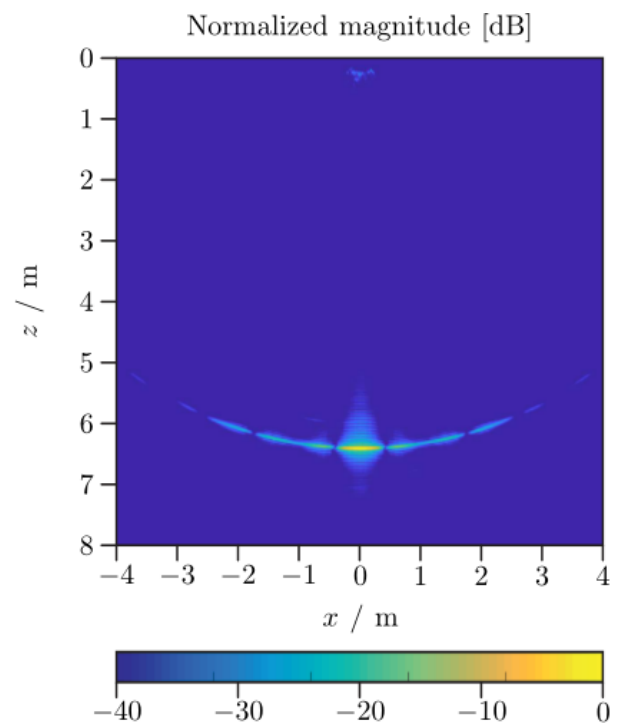

(a)

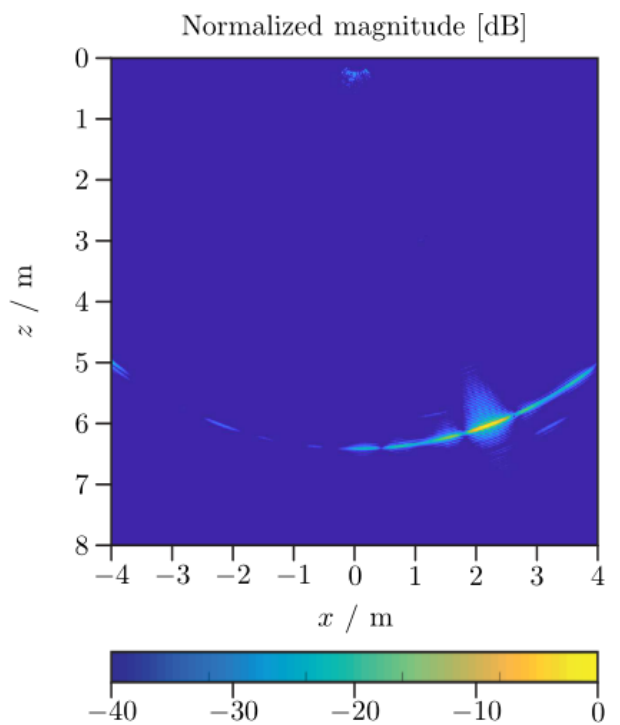

(b)

Figure 7. Radar images in a cross section $y=0$ of a corner reflector positioned at two different aspect angles: (a) $\theta_{1}=0^{\circ}$ and (b) $\theta_{2}=20^{\circ}$.

Figure 6 shows that the grating lobes in the transmit array factor $\mathrm{AF}_{\mathrm{Tx}}$ are suppressed by the zeros of receive array factor $\mathrm{AF}_{\mathrm{Rx}}$ and vice versa.

The resulting virtual array factor $\mathrm{AF}_{\mathrm{V}}$ shows no disturbing grating lobes any more, and an angular resolution $\Delta \theta=3.1^{\circ}$ and a side lobe suppression $\mathrm{SLS}=12.6 \mathrm{~dB}$, is achieved.

\section{Measurements}

The range resolution $\Delta R$, the angular resolution $\Delta \theta$ and a side lobe suppression SLS of the proposed MIMO radar system have been evaluated in a single target scenario. Therefore, a triangular corner reflector with a edge length of $25 \mathrm{~cm}$ has been positioned in an anechoic chamber at a range of $6.4 \mathrm{~m}$ for two different aspect angles $\theta_{1}=0^{\circ}$ and $\theta_{2}=20^{\circ}$.
A cross section of the corresponding radar images are shown in Fig. 7, achieving a range resolution $\Delta R=36 \mathrm{~mm}$.

Figure 8 shows the radar images in a polar cross section in order to evaluate the side lobe pattern at the target range $R=$ $6.4 \mathrm{~m}$.

For an aspect angle of $\theta_{1}=0^{\circ}$ an angular resolution $\Delta \theta=$ $3.1^{\circ}$ and a side lobe suppression SLS $=12.6 \mathrm{~dB}$ are achieved. As as expected, side lobe pattern corresponds to the virtual array factor $\mathrm{AF}_{\mathrm{V}}$, see Fig. 6c. For an aspect angle of $\theta_{1}=20^{\circ}$, the side lobe suppression SLS is reduced by $2.1 \mathrm{~dB}$ and the angular resolution $\Delta \theta$ is increased to $3.4^{\circ}$. This is mainly caused by the radiation pattern of the patch antenna elements and leads to a minor degradation in the measurement performance for higher aspects angles $\theta$. 


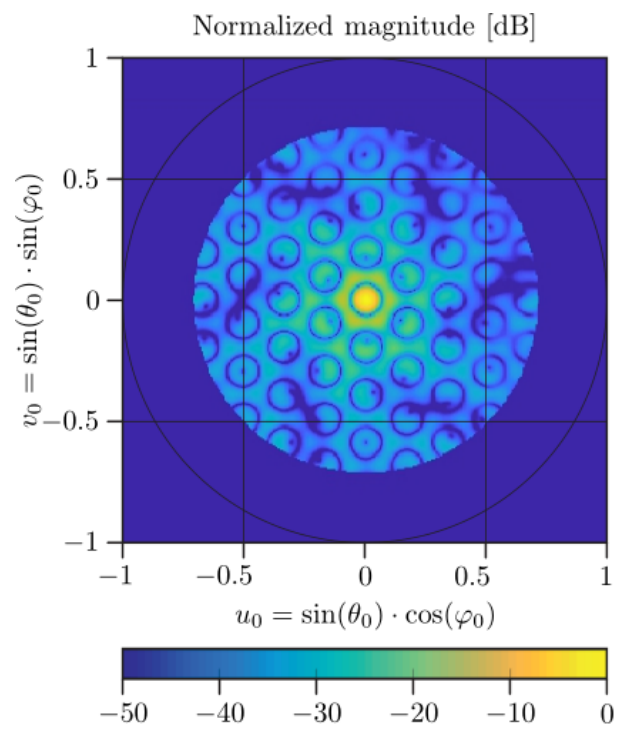

(a)

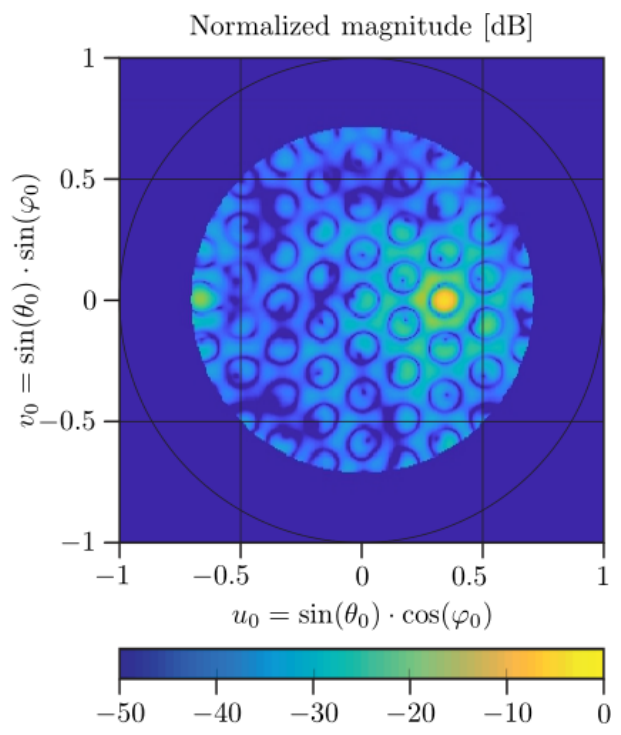

(b)

Figure 8. Radar images in a polar cross section $R=6.4 \mathrm{~m}$ of a corner reflector positioned at two different aspect angles: (a) $\theta_{1}=0^{\circ}$ and (b) $\theta_{2}=20^{\circ}$.

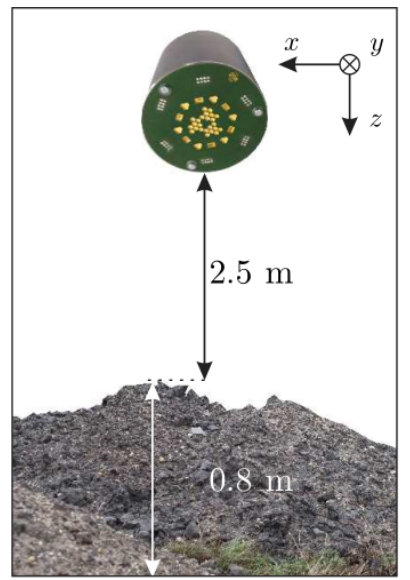

(a)

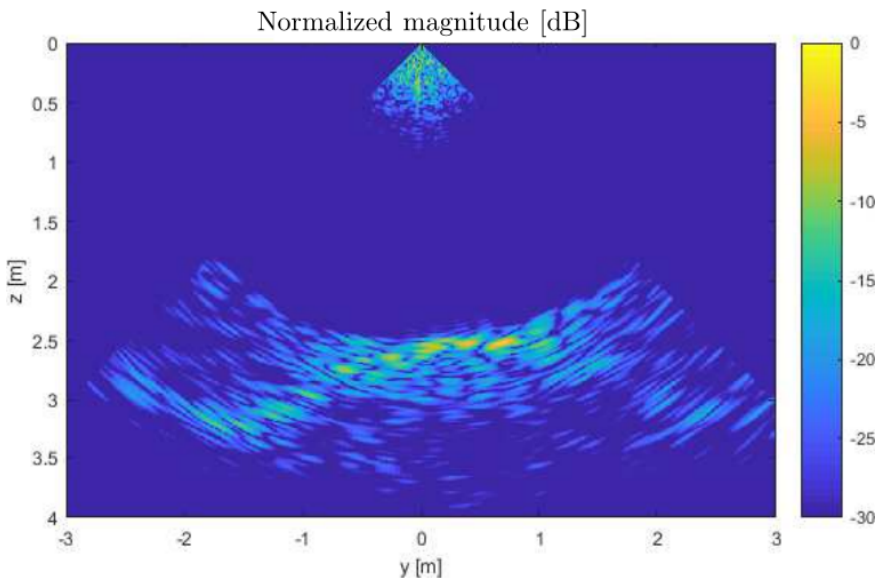

(b)

Figure 9. (a) Measurement scenario and (b) corresponding radar image in a vertical cross section $x=0$.

The proposed MIMO radar system has been evaluated in a realistic measurement scenario, consisting of a sand heap with a height of $0.8 \mathrm{~m}$. As shown in Fig. 9a, the radar system has been mounted $2.5 \mathrm{~m}$ above the heap and the echo data from all transmit and receive combinations has been processed to reconstruct 3D radar images. In Fig. 9b, a vertical cross section is shown, allowing for reconstruction of the heap. The crosstalk between the antenna elements along with the wave propagation across the printed circuit board are causing clutter in the radar image for ranges below $50 \mathrm{~cm}$.

\section{Conclusions}

In this paper, the design of a MIMO radar system with 18 transmit and 24 receive antennas has been presented. It has been shown that array topologies based on space filling fractals can be utilized to approximate a virtual aperture with a circular shape and to improve the side lobe suppression as well as the angular resolution. The performance of a sparse fractal MIMO array has been proven in a typical scenario, resulting in an improved accuracy for level measurement applications. 


\section{Appendix A: IWR1443 MIMO radar chip}

The Texas Instruments IWR1443 is a integrated MIMO radar chip realized in a $45 \mathrm{~nm}$ RFCMOS process. It allows for a compact realization of MIMO radar systems by combining analog FMCW radar transceivers with digital signal processors. In a master slave configuration, the FMCW ramp can be distributed to multiple slave chips in $19.25-20.25 \mathrm{GHz}$ range. An additional pulse is emitted by the master in order to synchronize the ramp start time between the analog-to-digital converters (ADC). The specification of the IWR1443 radar chip according to the data sheet is shown in Table A1.

Table A1. IWR1443 specification.

\begin{tabular}{lr}
\hline Parameter & Value \\
\hline Frequency range & $77-81 \mathrm{GHz}$ \\
Transmit channels & 3 \\
Receive channels & 4 \\
Transmit power & $12 \mathrm{dBm}$ \\
Receiver gain & $48 \mathrm{~dB}$ \\
Noise figure & $15 \mathrm{~dB}$ \\
Phase noise at $1 \mathrm{MHz}$ & $-93 \frac{\mathrm{dBc}}{\mathrm{Hz}}$ \\
Maximum intermediate frequency & $15 \mathrm{MHz}$ \\
ADC resolution & $12 \mathrm{bit}$ \\
ADC complex sampling rate & $18.75 \mathrm{Msps}$ \\
\hline
\end{tabular}


Data availability. Data used in this paper is available upon request: Christoph Dahl (christoph.dahl@ rub.de).

Author contributions. CD elaborated the concept for the research subject, performed the measurements, and wrote the paper. IR and MV have been supervising the entire process and performed the final review.

Competing interests. The authors declare that they have no conflict of interest.

Disclaimer. Publisher's note: Copernicus Publications remains neutral with regard to jurisdictional claims in published maps and institutional affiliations.

Special issue statement. This article is part of the special issue "Kleinheubacher Berichte 2020".

Review statement. This paper was edited by Madhu Chandra and reviewed by two anonymous referees.

\section{References}

Ahmed, S., Schiess, A., and Schmidt, L.: Near field mm-wave imaging with multistatic sparse 2d-arrays, in: European Radar Conference (EuRAD), 180-183, 2009.

Brooker, G., Scheding, S., Maclean, A., Hennessy, R., Lobsey, C., and Widzyk-Capehart, E.: Millimetre Wave Radar Vision for the Mining Industry, in: IEEE Conference on Sensors, https://doi.org/10.1109/icsens.2007.355472, 2006.

Buck, A. and Pozar, D.: Aperture-coupled microstrip antenna with a perpendicular feed, Electron. Lett., 22, 125, https://doi.org/10.1049/el:19860087, 1986.

Dahl, C., Vogt, M., and Rolfes, I.: A two-dimensional radar simulator for level measurement of bulk material in silos, in: German Microwave Conference (GeMiC), 221-224, https://doi.org/10.1109/gemic.2015.7107793, 2015a.

Dahl, C., Vogt, M., and Rolfes, I.: Comparison of virtual arrays for mimo radar applications based on hexagonal configurations, in: European Radar Conference (EuRAD), 417-420, https://doi.org/10.1109/EuRAD.2015.7346326, 2015b.

Dahl, C., Vogt, M., and Rolfes, I.: MIMO radar concepts based on antenna arrays with fractal boundaries, in: European Radar Conference (EuRAD), 41-44, 2016.
Dahl, C., Rolfes, I., and Vogt, M.: Investigation of fractal MIMO concepts for radar imaging of bulk solids, in: European Radar Conference (EURAD), 134-137, https://doi.org/10.23919/EURAD.2017.8249165, 2017a.

Dahl, C., Vogt, M., and Rolfes, I.: Fractal antenna arrays for MIMO radar applications, Int. J. Microw. Wirel. T., 9, 2019-2028, https://doi.org/10.1017/s1759078717001015, 2017b.

Ender, J. H. G. and Klare, J.: System architectures and algorithms for radar imaging by MIMO-SAR, in: IEEE Radar Conference, https://doi.org/10.1109/radar.2009.4976997, 2009.

Fishler, E., Haimovich, A., Blum, R., Chizhik, D., Cimini, L., and Valenzuela, R.: MIMO radar: an idea whose time has come, in: IEEE Radar Conference, https://doi.org/10.1109/nrc.2004.1316398, 2004.

Klare, J., Saalmann, O., Wilden, H., and Brenner, A. R.: Environmental monitoring with the imaging MIMO radars MIRA-CLE and MIRA-CLE X, in: IEEE International Geoscience and Remote Sensing Symposium, https://doi.org/10.1109/igarss.2010.5651824, 2010.

Nienhaus, K., Winkel, R., Mayer, W., Gronau, A., and Menzel, W.: An experimental study on using electronically scanning microwave radar systems on surface mining machines, in: IEEE Radar Conference, 509-512, https://doi.org/10.1109/RADAR.2007.374269, 2007.

Nienhaus, K., Hahn, M., and Winkel, R.: Wireless sensing applications in the mining and minerals industry, in: IEEE MTT-S International Microwave Workshop on Wireless Sensing, Local Positioning, and RFID, https://doi.org/10.1109/imws2.2009.5307897, 2009.

Volakis, J.: Antenna engineering handbook, John L. Volakis 2007, McGraw-Hill Education, ISBN: 9780071475747, chap. 3, p. 3, 2007.

Werner, D., Kuhirun, W., and Werner, P.: The peano-gosper fractal array, IEEE T. Antenn. Propag., 51, 2063-2072, https://doi.org/10.1109/TAP.2003.815411, 2003.

Werner, D., Kuhirun, W., and Werner, P.: Fractile arrays: a new class of tiled arrays with fractal boundaries, IEEE T. Antenn. Propag., 52, 2008-2018, https://doi.org/10.1109/TAP.2004.832327, 2004.

Zankl, D., Schuster, S., Feger, R., Stelzer, A., Scheiblhofer, S., Schmid, C., Ossberger, G., Stegfellner, L., Lengauer, G., Feilmayr, C., Lackner, B., and Burgler, T.: Blastdar - A large radar sensor array system for blast furnace burden surface imaging, IEEE Sens. J., 15, 5893-5909, https://doi.org/10.1109/JSEN.2015.2445494, 2015.

Zhuge, X. and Yarovoy, A.: Study on two-dimensional sparse mimo uwb arrays for high resolution near-field imaging, IEEE T. Antenn. Propag., 60, 4173-4182, https://doi.org/10.1109/TAP.2012.2207031, 2012. 